\title{
Equiseparability on Terminal Wiener Index
}

\author{
Xiaotie Denga ${ }^{\mathrm{a}, \mathrm{b}, 1}$, Jie Zhang ${ }^{\mathrm{b}, \mathrm{c}, *}$ \\ ${ }^{a}$ School of Information Science and Technology, Xiamen University, China. \\ ${ }^{b}$ Department of Computer Science, City University of Hong Kong, Hong Kong. \\ ${ }^{c}$ Computer Science Department, Aarhus University, Denmark.
}

\begin{abstract}
The aim of this paper is to explore the properties of terminal Wiener index, which is recently proposed by Gutman et al [3], and show the fact that there exist pairs of trees and chemical trees which can not be distinguished by it. We give some general methods to construct equiseparable pairs and compare the methods in the case of Wiener index. More specifically, we show that terminal Wiener index is degenerative to some extent.
\end{abstract}

Keywords: Equiseparability, terminal Wiener index, chemical tree.

\section{Introduction}

There are many chemical indices proposed as molecular-structure descriptors until now, one of the oldest and well studied chemical index is the Wiener index which was given by Wiener [4] in 1947. It can be expressed as:

$$
W(G)=\sum_{1 \leq i<j \leq n} d\left(v_{i}, v_{j}\right)
$$

where $d\left(v_{i}, v_{j}\right)$ is the distance between vertices $v_{i}$ and $v_{j}$ in a graph $G$, the summation goes over all pairs of vertices of the given graph. For trees, Wiener

\footnotetext{
*Corresponding author.The author acknowledges support from the Danish National Research Foundation and The National Science Foundation of China (under the grant 61061130540) for the Sino-Danish Center for the Theory of Interactive Computation, within which part of this work was performed.

Email address: jiezhang@cs.au.dk (Jie Zhang)

${ }^{1}$ This author is currently at University of Liverpool. Part of the work was conducted with the generous support of the Minjiang Scholar program, while visiting Xiamen University.
} 
got a very useful formula to calculate the Wiener index:

$$
W(T)=\sum_{e \in T} n_{1}(e \mid T) \cdot n_{2}(e \mid T)
$$

where $n_{1}(e \mid T)$ and $n_{2}(e \mid T)$ are the number of vertices of $T$, lying on the two sides of $e$. The summation on the right-hand side of the equation goes over all edges of the tree $T$. Obviously, if the tree $T$ has $n$ vertices, then for all of its edges,

$$
n_{1}(e \mid T)+n_{2}(e \mid T)=n
$$

Based on Wiener index, a general index called Variable Wiener Index has been proposed $[5,6]$ :

$$
W_{\lambda}(T)=\sum_{e \in T}\left[n_{1}(e \mid T) \cdot n_{2}(e \mid T)\right]^{\lambda}
$$

where $\lambda$ is an adjustable parameter.

Definition 1 [1]: Assuming $n_{1}(e \mid T) \leq n_{2}(e \mid T)$, two trees $T^{\prime}$ and $T^{\prime \prime}$ of order $n$ are said to be equiseparable if their edges $e_{1}^{\prime}, e_{2}^{\prime}, \cdots, e_{n-1}^{\prime}$ and $e_{1}^{\prime \prime}, e_{2}^{\prime \prime}, \cdots, e_{n-1}^{\prime \prime}$ can be labeled so that the equality $n_{1}\left(e_{i}^{\prime} \mid T^{\prime}\right)=n_{1}\left(e_{i}^{\prime \prime} \mid T^{\prime \prime}\right)$ holds for all $i=1,2, \cdots, n-1$.

Wiener index has been extensive used in Computational Biology, Preliminary screening of drugs and Complex Network. For example, it is a measurement of average distance in network $[7,8]$. In the design of economical networks, spanning trees of connected graph with smallest Wiener index are very important in practice [9]. In Chemistry, Wiener index measures the van der Waals surface area of an alkane molecule, which explains the correlations found between $W$ and a great variety of physico-chemical properties of alkanes [10]. But if two or more chemical trees are equiseparable, then those compounds will have similar physico-chemical properties which can not be distinguished by Wiener index. It is a main drawback of many chemical index structure-descriptors.

Gutman et al [1] pointed out that there does exist pairs of isomeric alkanes whose variable Wiener index coincide for all values of the parameter $\lambda$. Some former studies $[1,11]$ showed how to construct such equiseparable chemical trees. As another point of view, Vukičević and Gutman [2] gave a proof on almost all trees and chemical trees ${ }^{2}$ have equiseparable mates.

\footnotetext{
${ }^{2} \mathrm{~A}$ tree is a chemical tree if its maximum degree is at most 4 .
} 
In [12], Smolenskii et al made use of terminal distance matrices to encode molecular structures. The proposed reduced vector is less degenerative than some other molecular codes. Based on those applications, Gutman et al [3] proposed the concept of Terminal Wiener Index, which equals to the summation of distance between all pairs of pendent vertices ${ }^{3}$ of trees, i.e.

$$
T W(T)=\sum_{1 \leq i<j \leq k} d\left(v_{i}, v_{j}\right)
$$

where $v_{i}$ and $v_{j}$ are pendent vertices of tree $T, d\left(v_{i}, v_{j}\right)$ is the distance between them, and the sum goes over all pairs of such pendent vertices.

Similar to the proof of (2), Gutman got another way to calculate the terminal Wiener index.

$$
T W(T)=\sum_{e \in T} p_{1}(e \mid T) \cdot p_{2}(e \mid T)
$$

where $p_{1}(e \mid T)$ and $p_{2}(e \mid T)$ are the number of pendent vertices of $T$, lying on the two sides of $e$, and the summation embraces all the $n-1$ edges of $T$. We will use $p_{1}(e), p_{2}(e)$ instead of $p_{1}(e \mid T), p_{2}(e \mid T)$ when there is no confusion.

Similar to Wiener index, we define the variable terminal Wiener index so that it can have more molecular-structure descriptive power.

Definition 2: Variable terminal Wiener index is defined as follows:

$$
T W_{\lambda}(T)=\sum_{e \in T}\left[p_{1}(e) \cdot p_{2}(e)\right]^{\lambda}
$$

where $\lambda$ is an adjustable parameter.

Unfortunately, with this more powerful index, there still exist pairs of trees and chemical trees whose variable terminal Wiener index coincide for all values of the parameter $\lambda$. We can see it from the example in Figure 1, where $T_{1}$ and $T_{2}$ have same variable terminal Wiener index, $5 \cdot 2^{\lambda}$.

Based on this fact, we define the equiseparability w.r.t. terminal Wiener index.

Definition 3: Assuming $p_{1}(e) \leq p_{2}(e)$, two trees $T^{\prime}$ and $T^{\prime \prime}$ of order $n$ with the same number of pendent vertices are said to be equiseparable w.r.t. terminal Wiener index if their edges $e_{1}^{\prime}, e_{2}^{\prime}, \cdots, e_{n-1}^{\prime}$ and $e_{1}^{\prime \prime}, e_{2}^{\prime \prime}, \cdots, e_{n-1}^{\prime \prime}$ can

\footnotetext{
${ }^{3}$ In this paper, pendent vertices indicate leaves of the tree.
} 


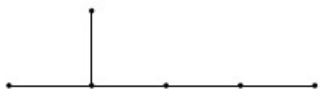

$T_{1}$

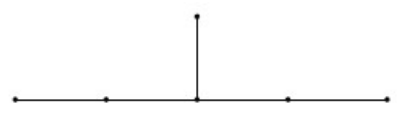

$T_{2}$

Fig.1

be labeled so that the equality $p_{1}\left(e_{i}^{\prime} \mid T^{\prime}\right)=p_{1}\left(e_{i}^{\prime \prime} \mid T^{\prime \prime}\right)$ holds for all $i=$ $1,2, \cdots, n-1$.

We see that equiseparable trees w.r.t. terminal Wiener index have equal terminal Wiener indices, variable terminal Wiener indices (for all $\lambda$ ).

In Section 2, we explore different rules for constructing equiseparable trees w.r.t Wiener index and terminal Wiener index. In Section 3, we give a formal proof of the fact that terminal wiener index has the degenerative phenomenon as the wiener index.

\section{Rules for constructing equiseparable trees with respect to ter- minal Wiener index}

First, we show that the methods of constructing equiseparable trees w.r.t Wiener index in $[1,11]$ can be extended to construct equiseparable trees w.r.t terminal Wiener index.

In [1], Gutman got some rules for constructing equiseparable chemical trees w.r.t. Wiener index. But they are in fact special cases of the method obtained in [11], which can be stated as:

Theorem 1 [11]: Let $T, X$ and $Y$ be arbitrary trees, each with more than two vertices. Let the tree $T_{1}$ be obtained from $T$ by identifying the vertices $u$ and $s$, and by identifying the vertices $v$ and $t$. Let $T_{2}$ be obtained from $T$ by identifying the vertices $u$ and $t$, and by identifying the vertices $v$ and $s$. Then if $X$ and $Y$ have equal number of vertices, the trees $T_{1}$ and $T_{2}$ are equiseparable. See Fig.2.

If we revise the condition felicitously, then Theorem 1 can be extended to construct equiseparable trees w.r.t. terminal Wiener index.

Theorem 2: Let trees $T_{1}$ and $T_{2}$ be constructed the same as they are in Fig.2. If $p_{x}-p_{s}=p_{y}-p_{t}$, then the trees $T_{1}$ and $T_{2}$ are equiseparable w.r.t. terminal Wiener index. $p_{x}$ and $p_{y}$ denote the number of pendent vertices of fragments $X$ and $Y$, respectively. $p_{s}$ is equal to 1 if $s$ is a pendent vertex of $X$, otherwise it is equal to $0 . p_{t}$ is defined similar to $p_{s}$. 

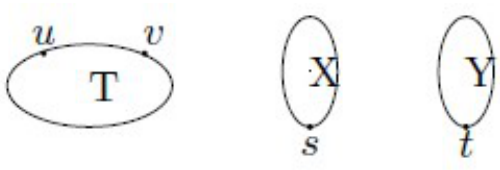

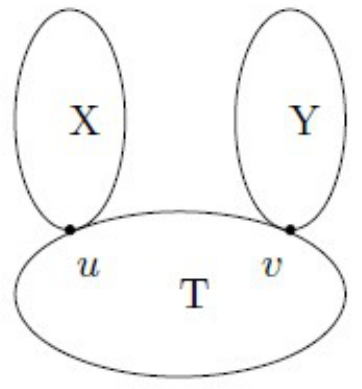

$T_{1}$

Fig.2

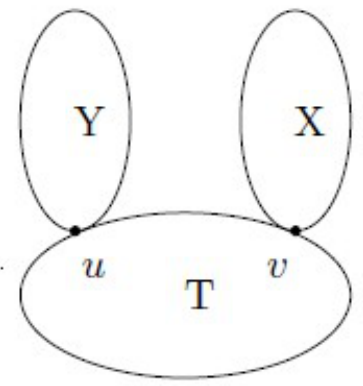

$T_{2}$

Proof: We prove it by classifying the edges of $T_{1}$ and $T_{2}$ into four types and each type of edges satisfy Definition 3.

(1) For edges belonging to $T$, lying on the same side of $u$ and $v$. For example, edge $e^{\prime}$ of $T_{1}$ and $e^{\prime \prime}$ of $T_{2}$, both lying on the left of $u$. We have $p_{1}\left(e^{\prime} \mid T_{1}\right)=p_{1}\left(e^{\prime \prime} \mid T_{2}\right)=p_{1}(e \mid T), p_{2}\left(e^{\prime} \mid T_{1}\right)=p_{2}\left(e^{\prime \prime} \mid T_{2}\right)=p_{2}(e \mid T)+p_{x}+p_{y}-k$, where $k$ is a constant which equals the number of pendent vertices among $\{u, v, s, t\}$. So this type of edges can be labeled so that $p_{1}\left(e^{\prime} \mid T_{1}\right)=p_{1}\left(e^{\prime \prime} \mid T_{2}\right)$ always holds. The same applies to edges lying on the right of $v$.

(2) For edges belonging to $X$. Obviously there is a bijection between the edges of fragment $X$ of $T_{1}$ and the edges of fragment $X$ of $T_{2}$, so this type of edges can also be labeled so that $p_{1}\left(e^{\prime} \mid T_{1}\right)=p_{1}\left(e^{\prime \prime} \mid T_{2}\right)$ always holds.

(3) For edges belonging to $Y$. It is the same as case (2).

(4) For edges belonging to $T$, lying between the vertices $u$ and $v$. According to whether vertices $u, v, s, t$ are pendent vertices of their corresponding fragments, this case can be divided into $2^{4}=16$ subcases. We only check three typical subcases here, others can be proved similarly.

(4.1) None of them is pendent vertex.

Then we have $p_{1}\left(e^{\prime} \mid T_{1}\right)=p_{1}(e \mid T)+p_{x}, p_{2}\left(e^{\prime} \mid T_{1}\right)=p_{2}(e \mid T)+p_{y}$ and $p_{1}\left(e^{\prime \prime} \mid T_{2}\right)=p_{1}(e \mid T)+p_{y}, p_{2}\left(e^{\prime \prime} \mid T_{2}\right)=p_{2}(e \mid T)+p_{x}$. Combined with $p_{s}=0$ and $p_{t}=0$, we get that the equality $p_{x}-p_{s}=p_{y}-p_{t}$ implies the edges lying between $u$ and $v$ satisfy Definition 3 . 


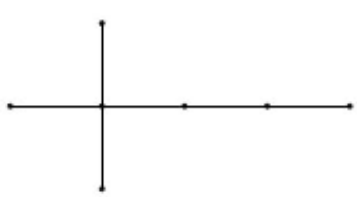

$T_{1}$

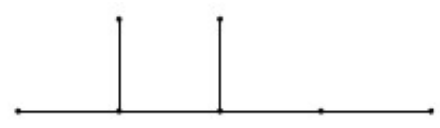

$T_{2}$

Fig. 3

(4.2) One of them is pendent vertex, for example, $s$ is a pendent vertex of $X$.

Then we have $p_{1}\left(e^{\prime} \mid T_{1}\right)=p_{1}(e \mid T)+p_{x}-1, p_{2}\left(e^{\prime} \mid T_{1}\right)=p_{2}(e \mid T)+p_{y}$ and $p_{1}\left(e^{\prime \prime} \mid T_{2}\right)=p_{1}(e \mid T)+p_{y}, p_{2}\left(e^{\prime \prime} \mid T_{2}\right)=p_{2}(e \mid T)+p_{x}-1$. Combined with $p_{s}=1$ and $p_{t}=0$, we get that the equality $p_{x}-p_{s}=p_{y}-p_{t}$ implies the edges lying between $u$ and $v$ satisfy Definition 3 .

(4.3) Two of them are pendent vertices, for example, $s$ is a pendent vertex of $X$ while $v$ is a pendent vertex of $T$.

Then we have $p_{1}\left(e^{\prime} \mid T_{1}\right)=p_{1}(e \mid T)+p_{x}-1, p_{2}\left(e^{\prime} \mid T_{1}\right)=p_{2}(e \mid T)+p_{y}-1$ and $p_{1}\left(e^{\prime \prime} \mid T_{2}\right)=p_{1}(e \mid T)+p_{y}, p_{2}\left(e^{\prime \prime} \mid T_{2}\right)=p_{2}(e \mid T)+p_{x}-2$. Combined with $p_{s}=1$ and $p_{t}=0$, we get that the equality $p_{x}-p_{s}=p_{y}-p_{t}$ implies the edges lying between $u$ and $v$ satisfy Definition 3 .

After checking all 16 subcases we get that edges lying between $u$ and $v$ can be labeled so that $p_{1}\left(e^{\prime} \mid T_{1}\right)=p_{1}\left(e^{\prime \prime} \mid T_{2}\right)$ always holds.

Aggregating these four cases, we can see that if $p_{x}-p_{s}=p_{y}-p_{t}$, then $p_{1}\left(e_{i}^{\prime} \mid T^{\prime}\right)=p_{1}\left(e_{i}^{\prime \prime} \mid T^{\prime \prime}\right)$ holds for all $i=1,2, \cdots, n-1$, which implies that trees $T_{1}$ and $T_{2}$ are equiseparable.

On the other hand, trees are equiseparable w.r.t. Wiener index does not imply they are equiseparable w.r.t. terminal Wiener index, since that the terminal Wiener index is the sum of the distance between all pairs of pendent vertices but not pairs of vertices. For example, the trees $T_{1}$ and $T_{2}$ in Fig.3 are equiseparable w.r.t. Wiener index but not equiseparable w.r.t terminal Wiener index. So, it is worth to find some general rules for constructing equiseparable trees w.r.t terminal Wiener index only.

The following theorem and corollary are rules to construct equiseparable trees w.r.t terminal Wiener index but not Wiener index.

Theorem 3: Let $Z$ be an arbitrary tree, $u$ is a vertex of $Z$, tree $T_{1}$ is obtained by identifying the vertices $u$ and $i, T_{2}$ is obtained by identifying the vertices $u$ and $j$. If $X$ and $Y$ have equal number of pendent vertices, then 


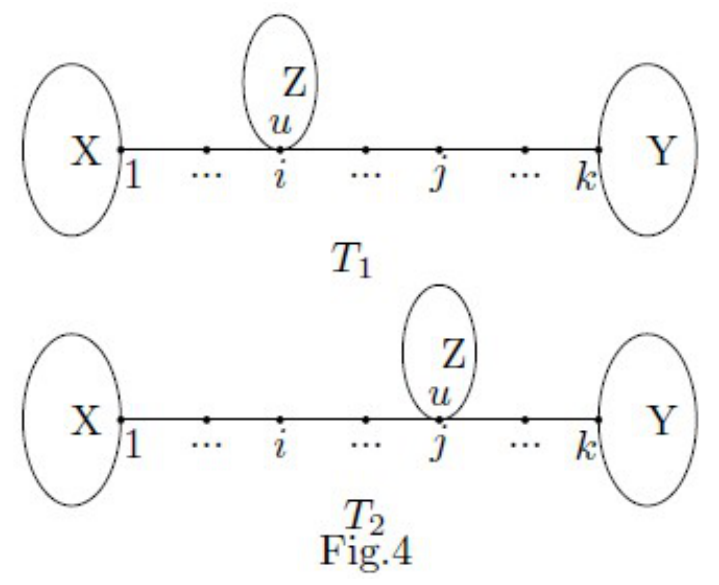

the trees $T_{1}$ and $T_{2}$ are equiseparable. See Fig. 4 .

Proof: Suppose the number of pendent vertices of fragments $X, Y$ and $Z$ are $p_{x}, p_{y}$ and $p_{z}$, respectively. If $u$ is a pendent vertex of $Z$ then $k$ is equal to 1 , otherwise $k$ is equal to 0 .

For each pair of edges $e^{\prime}$ of $T_{1}$ and $e^{\prime \prime}$ of $T_{2}$ which lying on the left of vertex $i$, the number of pendent vertices sit on the two sides of these edges are $p_{x}$ and $p_{y}+p_{z}-k$, respectively. So the edges lying on the left of vertex $i$ can be labeled so that $p_{1}\left(e_{i}^{\prime} \mid T_{1}\right)=p_{1}\left(e_{i}^{\prime \prime} \mid T_{2}\right)$ always holds.

The same applies to the edges which lying on the right of vertex $j$ and belonging to fragment $Z$, so we only need to consider the edges lying between vertices $i$ and $j$.

For the edge $e^{\prime}$ of $T_{1}$ which lying between $i$ and $j$, the number of pendent vertices sit on the two sides of $e^{\prime}$ are $p_{x}+p_{z}-k$ and $p_{y}$; For the edge $e^{\prime \prime}$ of $T_{2}$ which lying between $i$ and $j$, the number of pendent vertices sit on the two sides of $e^{\prime \prime}$ are $p_{x}$ and $p_{y}+p_{z}-k$.

Since $p_{x}=p_{y}$, we can label the edges $e_{1}^{\prime}, e_{2}^{\prime}, \cdots, e_{n-1}^{\prime}$ of $T_{1}$ and $e_{1}^{\prime \prime}, e_{2}^{\prime \prime}, \cdots, e_{n-1}^{\prime \prime}$ of $T_{2}$, so that the equality $p_{1}\left(e_{i}^{\prime} \mid T_{1}\right)=p_{1}\left(e_{i}^{\prime \prime} \mid T_{2}\right)$ holds for all $i=1,2, \cdots, n-1$. Therefore $T_{1}$ and $T_{2}$ are equiseparable w.r.t. terminal Wiener index.

Note that since $T W(T)$ only depends on the distance between pairs of pendent vertices, the position of fragment $Z$ can be arbitrary lying on the path from 1 to $k$. But for Wiener index, things are different. Fragments $X$ and $Y$ having equal number of vertices is not sufficient for equiseparability when fragment $Z$ moving arbitrary between vertex $i$ and $j$, we can see it from two trees in Fig.1.

Theorem 3 can be extended to the circumstances when they are more 

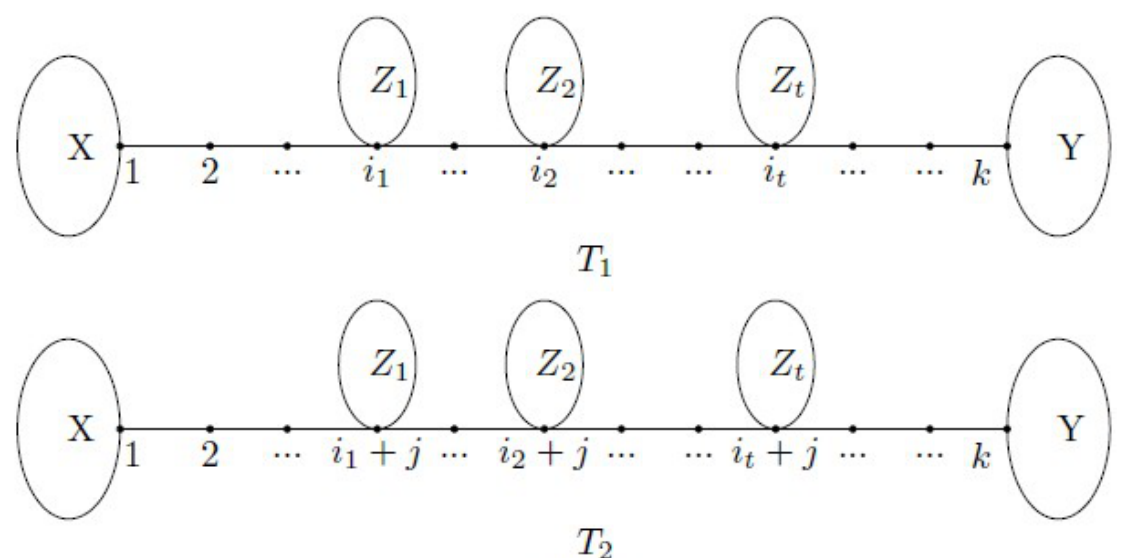

Fig.5

than one fragment on the path from 1 to $k$.

Corollary 1: If the fragments $X$ and $Y$ have equal number of pendent vertices, the fragments $Z_{1}, Z_{2}, \cdots, Z_{t}$ moving without changing the distance between them, then the resulting two (chemical) trees are equiseparable w.r.t. terminal Wiener index. See Fig.5 for illustration.

The proof of Corollary 1 is omitted here.

\section{Degeneracy of terminal Wiener index}

Vukičević and Gutman [2] developed a powerful technic to prove almost all trees and chemical trees have equiseparable mates w.r.t. Wiener index, the proof of the chemical trees case is omitted since it is more complicated than the case of trees. In this section, we show that the terminal Wiener index is degenerative by proving almost all chemical trees have equiseparable mates.

Let $T$ be an $n$-vertex tree with $k(2 \leq k \leq n-1)$ pendent vertices, $e_{1}, e_{2}, \cdots, e_{n-1}$ are its edges. We can relate $T$ to a sequence $\varphi(T)$ as follows. Assume that for each edge $e_{i}$ of tree $T, p_{1}\left(e_{i} \mid T\right) \leq p_{2}\left(e_{i} \mid T\right)$. Let $t_{j}(T)$ among the numbers $p_{1}\left(e_{i} \mid T\right), i=1,2, \cdots, n-1$, be equal to $j, j=1, \ldots,\lfloor k / 2\rfloor$. In other words, $t_{j}(T)$ is the number of edges such that the number of pendent vertices lying on one side of $e_{i}$ that are less than the other side, is equal to $j$. Then the ordered $\lfloor k / 2\rfloor$-tuple of integers (either positive or zero)

$$
\varphi(T)=\left(t_{1}(T), t_{2}(T), \cdots, t_{\lfloor k / 2\rfloor}(T)\right)
$$


is called the separation sequence of $T$.

Remark: (i) The separation sequence $\varphi(T)=\left(t_{1}(T), t_{2}(T), \cdots, t_{\lfloor k / 2\rfloor}(T)\right)$ does not dependent on the labeling of the edges of $T$;

(ii) Since an $n$-vertex tree $T$ with $k$ pendent vertices has $n-1$ edges, $\sum_{j=1}^{\lfloor k / 2\rfloor} t_{j}(T)=n-1$;

(iii) Let $T_{1}$ and $T_{2}$ be two $n$-vertices trees with $k$ pendent vertices. By Definition 3, $T_{1}$ and $T_{2}$ are equiseparable if and only if $\varphi\left(T_{1}\right)=\varphi\left(T_{2}\right)$, i.e., two $n$-trees with the same number of pendent vertices are equiseparable if and only if their separation sequence coincide.

Theorem 4: The terminal Wiener index is degenerative in the sense that almost all chemical trees have equiseparable mates.

Proof: Let $C T_{n}$ be the set of chemical trees of order $n$, and $U_{n}$ and $C U_{n}$ the sets of trees and chemical trees of order $n$ having no equiseparable mates, respectively. Let $|S|$ denote the number of elements in set $S$. Then what we need to prove is

$$
\lim _{n \rightarrow \infty} \frac{\left|C U_{n}\right|}{\left|C T_{n}\right|}=0
$$

We first give an upper bound of $\left|C U_{n}\right|$. It is obviously that $\left|C U_{n}\right| \leq\left|U_{n}\right|$.

Let $U_{n, k}$ be the set of trees with $n$ vertices and $k$ pendent vertices having no equiseparable mates. Then $U_{n}=U_{n, 2} \cup U_{n, 3} \cup \cdots \cup U_{n,\lfloor k / 2\rfloor}$, and $\left|U_{n}\right| \leq$ $\sum_{k=2}^{n-1}\left|U_{n, k}\right|$.

Let $A$ be the set of ordered $\lfloor k / 2\rfloor$-tuples of nonnegative integers whose sum is equal to $n-1$. Then $|A|=\left(\begin{array}{c}\lfloor k / 2\rfloor+n-2 \\ \lfloor k / 2\rfloor-1\end{array}\right)$ according to the standard result of Combinatorics.

Now, let $\varphi: U_{n, k} \rightarrow A$ and $\varphi(T)$ is the separation sequence of $T$ for any $T \in U_{n, k}$. By Remark (iii), $\varphi$ is injective, and we have $\left|U_{n, k}\right| \leq|A|=$ $\left(\begin{array}{c}\lfloor k / 2\rfloor+n-2 \\ \lfloor k / 2\rfloor-1\end{array}\right)$.

So, we have an upper bound on $\left|U_{n}\right|$,

$$
\left|U_{n}\right| \leq \sum_{k=2}^{n-1}\left|U_{n, k}\right| \leq \sum_{k=2}^{n-1}\left(\begin{array}{c}
\lfloor k / 2\rfloor+n-2 \\
\lfloor k / 2\rfloor-1
\end{array}\right)
$$




$$
=\left\{\begin{aligned}
2 \sum_{k=1}^{(n-3) / 2}\left(\begin{array}{c}
k+n-2 \\
k-1
\end{array}\right) & +\left(\begin{array}{c}
\frac{n-1}{2}+n-2 \\
\frac{n-1}{2}-1
\end{array}\right) \text { for } n \text { is odd; } \\
& 2 \sum_{k=1}^{\frac{n}{2}-1}\left(\begin{array}{c}
k+n-2 \\
k-1
\end{array}\right) \text { for } n \text { is even }
\end{aligned}\right.
$$

by employing combinatorial recursive formula, we get

$$
\left|C U_{n}\right| \leq\left|U_{n}\right| \leq\left\{\begin{array}{c}
2 \sum_{k=1}^{(n-1) / 2}\left(\begin{array}{c}
k+n-2 \\
k-1
\end{array}\right) \\
2 \sum_{k=1}^{\frac{n}{2}-1}\left(\begin{array}{c}
k+n-2 \\
k-1
\end{array}\right)
\end{array} \leq\left\{\begin{array}{c}
2\left(\begin{array}{c}
\frac{3 n-3}{2} \\
\frac{n-3}{2}
\end{array}\right) \text { for } n \text { is odd; } \\
2\left(\begin{array}{c}
\frac{3 n-4}{2} \\
\frac{n-4}{2}
\end{array}\right) \text { for } n \text { is even }
\end{array}\right.\right.
$$

For $\left|C T_{n}\right|$, Otter [13] obtained an asymptotic value for the number of trees $T_{n, m}$ of order $n$ and maximum degree $m$, i.e., for $3 \leq m<\infty$,

$$
\left|T_{n, m}\right| \sim \frac{\beta^{3} \cdot a_{m-3}}{4 \sqrt{\pi} \cdot \alpha^{-2.5}} \frac{\alpha^{n}}{n^{2.5}}
$$

where $\alpha, \beta$ and $a_{m-3}$ are constant for any fixed $m$. Specifically, for $m=4$, i.e., for chemical trees, $\alpha=2.81546, \beta=3.08039, a_{1}=2.11742$. Hence we get

$$
\left|C T_{n}\right| \sim k \cdot \frac{\alpha^{n}}{n^{2.5}}, \quad k=0.65632, \quad \alpha=2.81546
$$

Obviously, $\left|C T_{n}\right|$ is exponential on $n$. Then we have

$$
\lim _{n \rightarrow \infty} \frac{\left|C U_{n}\right|}{\left|C T_{n}\right|}=0
$$

\section{References}

[1] I.Gutman, B.Arsić, B.Furtula, Equiseparable chemical trees, J. Serb. Chem. Soc., 68, 549-555 (2003).

[2] D.Vukičević, I.Gutman, Almost all trees and chemical trees have equiseparable mates. J. Comput. Chem. Jpn., 3(3), 109-112 (2004). 
[3] I.Gutman, B.Furtula, M.Petrović, Terminal Wiener index, J. Math. Chem. DOI: 10.1007/s10910-008-9476-2.

[4] H.Wiener, Structural determination of paraffin boiling points, J. Am. Chem. Soc, 69, 17-20 (1947).

[5] B.Lučić, A.Miličević, S.Nikolić, N.Trinajstić, On variable Wiener index, Indian J. Chem., 42A, 1279-1282 (2003).

[6] I.Gutman, D.Vukičević, J.Žerovnik, A class of modified Wiener indices, Croat. Chem. Acta, 77, 103-09 (2004).

[7] S.Wuchtya, P.F. Stadler, Centers of complex networks, Journal of Theoretical Biology., 223, 45-53 (2003).

[8] J. Zhao, H. Yu, J. Luo, Z. W. Cao and Y. Li, Complex networks theory for analyzing metabolic networks, Chinese Science Bulletin., DOI: 10.1007/s11434-006-2015-2.

[9] A. A. Dobrynin, R. Entringer and I. Gutman, Wiener Index of Trees: Theory and Applications, Acta Applicandae Mathematicae., 66, 211-249 (2001).

[10] I.Gutman, T.Körtvélyesi, Wiener indices and molecular surfaces, Z. Naturforsch., 50a, 669-671 (1995).

[11] I.Gutman, B.Furtula, D.Vukičević, B.Arsić, Equiseparable molecules and molecular graphs, Indian J.Chem. 43, 7-10 (2004).

[12] E.A.Smolenskii, E.V.Shuvalova, L.K.Maslova, I.V.Chuvaeva, M.S.Molchanova, Reduced matrix of topological distances with a minimum number of independent parameters: distance vectors and molecular codes, J. Math. Chem., DOI: 10.1007/s10910-008-9393-4.

[13] R.Otter, The number of trees, The Annals of Mathematics, Second Series, 49(3), 583-599 (1948). 